\title{
Towards Managing Digital Transformation in Manufacturing Industry: Theoretical Framework
}

\author{
Yasser Omar Abdallah a,c,1, Essam Shehab ${ }^{\mathrm{b}}$, Ahmed Al-Ashaab ${ }^{\mathrm{a}}$ \\ a Manufacturing Department, School of Aerospace, Transport \& Manufacturing, \\ Cranfield University, Cranfield, Bedford, UK \\ ${ }^{b}$ Mechanical and Aerospace Engineering Department, School of Engineering and \\ Digital Sciences, Nazarbayev University, Nur-Sultan, Kazakhstan \\ essam.shehab@nu.edu.kz,https://nu.edu.kz/faculty/essam-shehab \\ ${ }^{c}$ Business Administration Department, Faculty of Commerce, Mansoura University, \\ Mansoura, Egypt
}

\begin{abstract}
Although digital transformation has been extensively researched in recent years, there is no clear definition, especially in the manufacturing industry. In addition, there is no oblivious model for the current manufacturing firms to be digitally transformed. As a part of a current research project, this paper proposes a framework for the digital transformation process in the manufacturing industry to be used and applied by various firms, especially small and medium enterprises (SMEs). The proposed framework combines the four main aspects of Digital transfroamtion in an integrated way. These four areas are Peaple, Strategy and Leadership, Business Processes, Enabling Technologies and Tools. The framework balances between the management and technological aspects of Digital Transformation implementation inside the manufacturing organisation.
\end{abstract}

Keywords. Digital Transformation, Framework, Manufacturing

\section{Introduction}

There is no doubt that digital transformation is crucial for manufacturing companies to succeed and continue to operate in today's fierce competition. Moreover, concepts like Industry 4.0, Internet of Things (IoT), Cloud Computing, and many other new technologies can change business. Digital Transformation becomes an essential practice inside any manufacturing organisation in today's exponential change towards digital technologies and utilising these technologies in different fields and sectors regarding doing business in these fields. This affects the way of operations inside the organisation and affects the way of reaching the customer and providing the industry's goods and services ${ }^{1}$. As one of the most critical sectors inside any economy, Manufacturing

\footnotetext{
${ }^{1}$ Corresponding author, Manufacturing Department, School of Aerospace, Transport \& Manufacturing, Cranfield University, Cranfield, Bedford, UK Yasser.Abdallah@cranfield.ac.uk, https://www.cranfield.ac.uk/ people/yasser-abdallah-24604662
} 
Industry is greatly affected by the digitalisation of manufacturing processes by developing new technologies that can enhance the productivity of the operations processes inside any manufacturing organisation.

Digital Transformation (DT) in the manufacturing industry play a vital role in keeping the organisation in the race of competition. The only way of sustainability now inside any competitive market is by transforming your business processes into the digital world ${ }^{2}$. From here comes the importance of digital transformation of existing firms to keep the race with even the digital-native organisations that appeared in the past 10-15 years.

As part of an ongoing research project, this paper will propose a framework for understanding and implementing Digital Transformation inside the manufacturing industry. This research demonstrates the key steps and phases necessary to implement DT successfully with the manufacturing industry's focus.

\section{Definition of Digital Transformation}

According to the literature, few articles focused on defining what Digital Transformation is, especially in the manufacturing-focused papers, and there was an overlap between defining digital transformation and other related terms, for example, industry 4.0 or digital technologies ${ }^{3}$. From our analysis of the concept and after reviewing these definitions, we constructed a conceptual definition of digital transformation with the focus of the manufacturing industry as follow:

Digital Transformation (DT) is a customer-centric mechanism that enables continuous improvement in the productivity of the manufacturing processes using advanced digital technologies such as cloud computing, Internet of Things, big data analytics, digital twin in all aspects of the organisation.

From this proposed definition, we illustrate the critical elements of this definition as follows:

- Customer-centric element: most of the previous definitions neglect the customer's voice when talking about transformation processes. However, listening to the customer is crucial in formulating the organisation's digital transformation process to increase customer satisfaction and organisational profitability.

- Continuity element: Digital transformation is a process that never ends. If more technologies evolve and arise over time, we continually need to transform our business to cope with this technology advancement. So, it is not one-time planning and implementation; manufacturing firms need to understand that element remain competitive in the market.

- Digital technologies element: the technology element in the digital transformation is crucial, and selecting the most appropriate digital technologies that will adapt to your manufacturing theme is crucial in the digital transformation process's success.

- $\quad$ The organisation element: the digital transformation process will not succeed without embedding it into all aspects of the organisation. This requires integrating digital technologies into all aspects of the organisation to ensure that human factors in the firm make the best use of digital technologies to improve manufacturing processes' productivity. 


\section{Digital Transformation Framework}

Digital transformation is not about technology only as it requires integrating all parts of the organisation to develop and implement this transformation process successfully ${ }^{4}$. Moreover, to make it a continuous process, we need to change its strategy and embed this transformation into its core values, vision, and even culture. As in figure (1), we categorised the building blocks of Digital transformation as follows: People, Tools and Enabling Technology, Strategy and Leadership, Business Processes. This Framework is developed after analysing and categorising the digital transformation models and processes already existing in the literature and came up with a model that suits manufacturing indusry ${ }^{2,5}$.

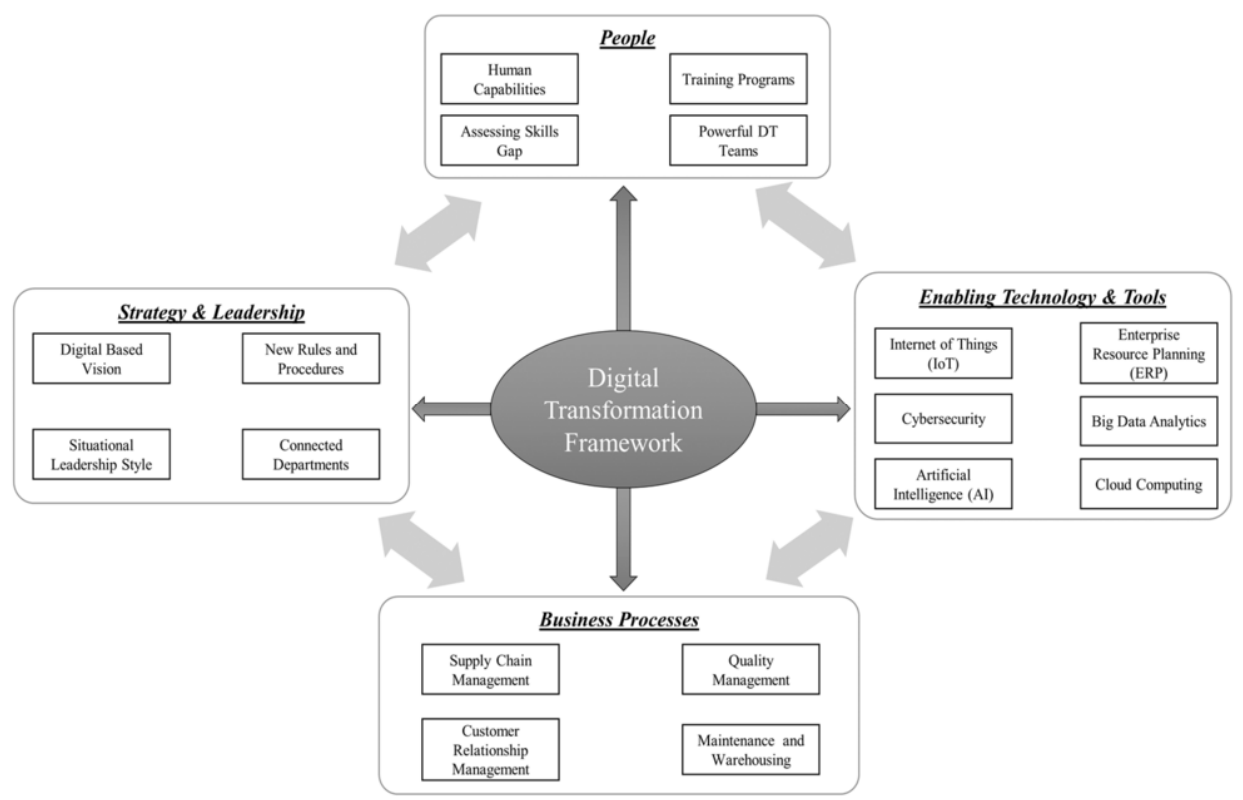

Figure 1 Digital Transformation Framework

\subsection{People}

Human capital is the backbone of any change projects inside the organisation. Digital Transformation requires special skills that need to be embedded in the employees and workers of the organisation. The first step to begin developing the organisation human capital is to assess the gap that existed in the digital skills in the organisation ${ }^{6}$.

Another crucial element in human capital is team building and management. Digital transformation is a group work that needs different departments and parts to be integrated to achieve good digital transformation results. The team should consist of 1 . The digital transformation leader manages the whole process and resolves any conflict during the project's implementation. 2. The change agent who encourages other members to cope with the change and be flexible to their job roles and the way of doing their work. 3.The business expert who has experience in marketing and distribution channels operations to reflect their perspective inside the project. 4.The data architect who analyses and 
generates reports that help the top management take decisions at the right time as this is a swift pace process that needs much flexibility and taking the right decisions at the right time. 5.The financial analyst who makes the cost-benefit analysis for the project and handles the project's financial budget. 6. The UX professional who can reflect the customer's voice and make the solutions the organisation provides suitable for the enduser $^{7}$.

\subsection{Strategy \& Leadership Style}

As highlighted before, in the proposed definition of DT, it is a continuous process that needs to be embedded in an organisation's strategy. Top management should create an organisational culture that encourages digital transformation practices and make internal incentives for these practices ${ }^{8}$.

The leader plays a significant role in the digital transformation. Changing their leadership style to suit the situation (Situational Leadership Style) is the most appropriate digital transformation project. A leader should have the following traits to be successful in the process: Flexibility: Being open to change requires an entrepreneurial attitude. Diversified Knowledge: By looking at what is going on in other sectors and seeing what is working and applicable to their industries. Priority and Results Focus: These are a must-win process that determines success or failure and are focused on improving the company's performance in the marketplace. Ownership and Responsibility: People respect courage and accountability. To lead effectively, executives and managers need to hold themselves responsible for their team's performance ultimately ${ }^{9}$.

\subsection{Enabling Technologies \& Tools}

One of the critical determinants of the Digital Transformation process's success is its digital technologies. Many researchers state different technologies used in the Digital Transformation process according to the organisation's field, and All these technologies are linked together to achieve a more valid Digital Transformation process

One of the most technologies that enable Digital Transformation inside any organisation is artificial intelligence and its various applications. With this technology and machine learning, equipment becomes more efficient and effective in their operations as they become more aware of the processes and how to self-learn and solve any problems issued inside the production processes ${ }^{10}$.

IoT technology enables businesses to link the physical world with the digital world. It helps manufacturing firms gather more data from machines and equipment, which helps understand the manufacturing problems and how to handle them more efficiently to make more productive operations.

Moreover, one of the essential elements in the successful implementation of Digital Transformation is its digital world's security. As mentioned earlier, With the increasing number of cyber-attacks each day, cybersecurity technology becomes vital in any organisation today. Manufacturing organisations need to build a robust cyber system to ease the accessibility of data and, at the same time, make it secure enough from digital attacks ${ }^{9}$.

Cloud Computing is also one of the foundations of Digital Transformation. With agile applications and the need to access data anytime, anywhere, the organisation needs to have a robust cloud system that enables employees to work with it and have the 
accessibility of data necessary to perform their operations. Machine learning and IoT provide vast amounts of data about everything that happened in the factory. Big data analytics help provide great insights about these data and how to collect, analyse and organise these amounts of data to help the management take the right decisions promptly and provide new ways of innovation ${ }^{11}$.

Robots is one of the advanced technologies used inside the manufacturing firms to handle complex tasks to reduce worker burnout and increase the factory's health and safety procedures. However, the manufacturing organisation must keep the right balance between robots and human power to ensure the processes' agility and make the most of the firm machines.

ERP is a software toolkit that helps the organisation link all its various departments to help transfer the information vastly and in real-time. Cloud ERP will be more efficient in data privacy and data transfer velocity than traditional ERP software with a robust cloud system $^{12}$.

\subsection{Business Processes}

Linking departments together is very important to digital transformation. Connected business processes are vital, from supply chain integration with suppliers and their different tiers to warehousing and quality management and ending with customer relationship management and hearing the customer feedback. Linking all the processes across the value chain is very important as single efforts will not succeed without integrating other processes across different departments.

This complexity of suppliers should be managed jointly for the transportation and storage of raw materials through Supplier Relationship Management software using Internet of Things and Big Data analytics to ensure that there will be no shortage of raw materials for the manufacturing processes. So, this needs to be carefully managed and coordinated with the procurement department.

Using advanced Digital Transformation technologies in the organisation's manufacturing process like robots/cobots through advanced robotics management will enhance the organisation's productivity and continuously develop its products and services

\section{Conclusions}

This paper proposes a theoretical framework for digital transformation implementation inside the manufacturing organisation. The model proposed four critical elements that should be integrated to achieve successful digital transformation practices. These elements are People, Strategy and Leadership, Technologies, Business Processes.

The results and issues raised in this paper provide a valuable basis for further work to be focused upon. Digital Transformation is a concept of enormous potential significance for manufactures. It offers routes for companies to step up the value chain and leverage value-added business activities.

\section{Acknowledgement}

The authors would like to thank the Egyptian Government for funding this research under the umbrella of the ministry of Higher Education grants program for PhD students. 


\section{References}

[1] Fernández-Rovira C, Álvarez Valdés J, Molleví G, Nicolas-Sans R. The digital transformation of business. Towards the datafication of the relationship with customers. Technological Forecasting and Social Change. 2021;162:120339. doi:https://doi.org/10.1016/j.techfore.2020.120339

[2] Llopis-Albert C, Rubio F, Valero F. Impact of digital transformation on the automotive industry. Technological Forecasting and Social Change. 2021;162:120343. doi:https://doi.org/10.1016/j.techfore.2020.120343

[3] Culot G, Nassimbeni G, Orzes G, Sartor M. Behind the definition of Industry 4.0: Analysis and open questions. International Journal of Production Economics. 2020;226:107617. doi:https://doi.org/10.1016/j.ijpe.2020.107617

[4] Benitez GB, Ayala NF, Frank AG. Industry 4.0 innovation ecosystems: An evolutionary perspective on value cocreation. International Journal of Production Economics. 2020;228:107735. doi:https://doi.org/10.1016/j.ijpe.2020.107735

[5] Götz M, Jankowska B. Adoption of industry 4.0 technologies and company competitiveness: Case studies from a post-transition economy. Foresight and STI Governance. 2020;14(4):6178. doi:10.17323/2500-2597.2020.4.61.78

[6] Brock JKU, von Wangenheim F. Demystifying Ai: What digital transformation leaders can teach you about realistic artificial intelligence. California Management Review. 2019;61(4):110-134. doi:10.1177/1536504219865226

[7] Guinan PJ, Parise S, Langowitz N. Creating an innovative digital project team: Levers to enable digital transformation. Business Horizons. 2019;62(6):717-727. doi:10.1016/ J.BUSHOR.2019.07.005

[8] Ilvonen I, Thalmann S, Manhart M, Sillaber C. Reconciling digital transformation and knowledge protection: A research agenda. Knowledge Management Research and Practice. 2018;16(2):235-244. doi:10.1080/14778238.2018.1445427

[9] Warner KSR, Wäger M. Building dynamic capabilities for digital transformation: An ongoing process of strategic renewal. Long Range Planning. 2019;52(3):326-349. doi:10.1016/j.lrp.2018.12.001

[10] Hartley JL, Sawaya WJ. Tortoise, not the hare: Digital transformation of supply chain business processes. Business Horizons. 2019;62(6):707-715. doi:10.1016/J.BUSHOR. 2019.07.006

[11] Borangiu T, Trentesaux D, Thomas A, Leitão P, Barata J. Digital transformation of manufacturing through cloud services and resource virtualisation. Computers in Industry. 2019;108:150-162. doi:10.1016/j.compind.2019.01.006

[12] Ananyin V, Zimin K, Lugachev M, Gimranov R, Skripkin K. Digital organisation: Transformation into the new reality. Business Informatics. 2018;2018(2):45-54. doi:10.17323/1998-0663.2018.2.45.54 